\title{
Assessing the calibration in toxicological in vitro models with conformal prediction
}

\author{
Andrea Morger ${ }^{1}$, Fredrik Svensson ${ }^{2}$, Staffan Arvidsson McShane ${ }^{3}$, Niharika Gauraha ${ }^{3,4}$, Ulf Norinder ${ }^{3,5,6}$, \\ Ola Spjuth ${ }^{3 \dagger}$ and Andrea Volkamer ${ }^{1 *+}+(0)$
}

\begin{abstract}
Machine learning methods are widely used in drug discovery and toxicity prediction. While showing overall good performance in cross-validation studies, their predictive power (often) drops in cases where the query samples have drifted from the training data's descriptor space. Thus, the assumption for applying machine learning algorithms, that training and test data stem from the same distribution, might not always be fulfilled. In this work, conformal prediction is used to assess the calibration of the models. Deviations from the expected error may indicate that training and test data originate from different distributions. Exemplified on the Tox21 datasets, composed of chronologically released Tox21Train, Tox21Test and Tox21Score subsets, we observed that while internally valid models could be trained using cross-validation on Tox21Train, predictions on the external Tox21Score data resulted in higher error rates than expected. To improve the prediction on the external sets, a strategy exchanging the calibration set with more recent data, such as Tox21Test, has successfully been introduced. We conclude that conformal prediction can be used to diagnose data drifts and other issues related to model calibration. The proposed improvement strategy-exchanging the calibration data only-is convenient as it does not require retraining of the underlying model.
\end{abstract}

Keywords: Toxicity prediction, Conformal prediction, Data drifts, Applicability domain, Calibration plots, Tox21 datasets

\section{Introduction}

Machine learning (ML) methods are ubiquitous in drug discovery and toxicity prediction $[1,2]$. In silico toxicity prediction is typically used to guide toxicity testing in early phases of drug design [3]. With more high-quality standardised data available, the (potential) impact of ML methods in regulatory toxicology is growing [4]. The collection of available toxicity data is increasing, thanks in part to high-throughput screening programs such as ToxCast [5] and Tox21 [6, 7], but also with public-private partnerships such as the eTOX and eTRANSAFE projects, which focus on the sharing of (confidential) toxicity

\footnotetext{
${ }^{*}$ Correspondence: andrea.volkamer@charite.de

${ }^{\dagger}$ Ola Spjuth and Andrea Volkamer-Shared senior authorship

${ }^{1}$ In Silico Toxicology and Structural Bioinformatics, Institute of Physiology, Charité Universitätsmedizin, Berlin, Germany

Full list of author information is available at the end of the article
}

data and ML models across companies [8, 9]. In any case, no matter which underlying data and ML method is used, it is essential to know or assess if the ML model can be reliably used to make predictions on a new dataset.

Hence, validation of ML models is crucial to assess their predictivity. Several groups investigated random vs. rational selection of optimal test/training sets, e.g. using cluster- or activity-based splits, with the goal of better reflecting the true predictive power of established models [10-14]. Martin et al. [11] showed that rational selection of training and test sets-compared to random splitsgenerated better statistical results on the (internal) test sets. However, the performance of both types of regression models on the-artificially created-external evaluation set was comparable.

Thus, further metrics to define the applicability domain (AD), the domain in which an ML classifier can reliably

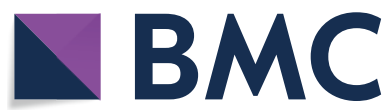

(c) The Author(s) 2021. This article is licensed under a Creative Commons Attribution 4.0 International License, which permits use, sharing, adaptation, distribution and reproduction in any medium or format, as long as you give appropriate credit to the original author(s) and the source, provide a link to the Creative Commons licence, and indicate if changes were made. The images or other third party material in this article are included in the article's Creative Commons licence, unless indicated otherwise in a credit line to the material. If material is not included in the article's Creative Commons licence and your intended use is not permitted by statutory regulation or exceeds the permitted use, you will need to obtain permission directly from the copyright holder. To view a copy of this licence, visit http://creativeco mmons.org/licenses/by/4.0/. The Creative Commons Public Domain Dedication waiver (http://creativecommons.org/publicdomain/ zero/1.0/) applies to the data made available in this article, unless otherwise stated in a credit line to the data. 
be applied [15-21], are needed. Besides traditional metrics accounting for chemical space coverage, Sheridan [20] discussed uncertainty prediction regression models, fitted with the activity prediction errors as labels and diverse $\mathrm{AD}$ metrics as descriptors (e.g. accounting for variation among RF tree predictions, predicted activity ranges with different confidence, or similarity to nearest neighbours). Since in classification models, the response/ activity is a categorical value, only the chemical space remains to define the AD. Mathea et al. [15] categorised the available methods into novelty and confidence estimation techniques. The former consider the fit into the underlying chemical descriptor space as a whole, whereas the latter focus on the reliability of predictions, i.e. data points may be well embedded in the descriptor space but abnormal regarding their class label.

A popular method for confidence estimation is conformal prediction $(\mathrm{CP})$, which has in recent years been widely applied in the drug discovery and toxicity prediction context $[15,22]$. In CP, ML models are trained, and with the help of an additional calibration set (inductive conformal prediction [23]), the predictions are calibrated, i.e. ranked based on previously seen observations, resulting in so-called conformal p-values or simply p-values (not to be confused with statistical p-values from hypothesis testing). The design of the CP statistical framework guarantees that the error rate of the predictions will not exceed a user-specified significance level. The control of this significance level makes $\mathrm{CP}$ advantageous compared to traditional confidence estimation methods, such as distance from the decision boundary, or ensemble models [15].

ML algorithms rely on the assumption that the probability distribution of the training data and test data are I.I.D. (independent and identically distributed). For conformal prediction, a slightly weaker assumption in the form of exchangeability is assumed for producing well-calibrated models [24]. This assumption is nevertheless not always fulfilled, especially when training and test data come from different sources. For example, data drifts were observed between training and test data of the USPS (handwritten digits) and the Statlog Satellite (satellite image) datasets [25]. Similar observations were made in the toxicity prediction context when applying androgen receptor agonism CP models trained on publicly available data to an industrial dataset [26]. Some efforts to look at data exchangeability include studies using martingales to uncover exchangeability issues in an online setting [25].

In this work, we explored how the above described concepts of conformal prediction can be used to assess the quality of the model calibration when trained and applied on various toxicological in vitro datasets or subsets. For this purpose, the freely available Tox 21 datasets [27], initially prepared for a data challenge to encourage model building and benchmarking toxicity prediction, were used. We show that conformal prediction allows us to identify data drifts between the Tox 21 datasets, and we also propose strategies to mitigate this.

\section{Data and methods}

In this section, first the used Tox 21 datasets are introduced. Second, the general conformal prediction framework along with aspects such as aggregation, evaluation and strategies to improve the calibration are described. Finally, the set-up and the individual computational experiments of this work are explained, including a reference to code and data availability.

\section{Data collection, preprocessing and encoding Tox21 datasets}

The investigations in this work were performed on the freely available Tox 21 datasets [27]. They consist of approximately 10,000 chemicals, which were tested on up to 12 endpoints of the nuclear receptor (NR) and stress response (SR) pathways. As the dataset was released in a challenge setting, the three subsets were chronologically published to the Tox 21 Data Challenge participants: Tox21Train for training the models, Tox21Test as an intermediate set for the leaderboard to check the performance (and for participants to improve their models), and Tox21Score as the final dataset to determine the best performing models. The respective datasets were downloaded from the US National Center for Advancing Translational Sciences [28] on January 29th, 2019. Each compound was provided in sdf-format together with a binary value (0/non-toxic, or $1 /$ toxic) for each of the 12 endpoints ( $X$ if no assay outcome was available for the compound). Note that throughout this manuscript, the Tox 21 datasets are, consistently, referred to as Tox21Train, Tox21Test and Tox21Score, this should not be confused with additional training and test set splits necessary for the ML/CP model set-ups.

\section{Data preprocessing}

The datasets were standardised as described in Morger et al. [26]. Briefly, the IMI eTox standardiser tool was applied to discard non-organic compounds, to exert certain structure standardisation rules, to neutralise, and to remove salts [29]. Before and after applying the standardisation protocol, compounds with duplicate InChIs (IUPAC International Chemical Identifiers [30]) but disagreeing labels were discarded. Furthermore, remaining mixtures and fragments with less than four heavy atoms were removed. The numbers of data points available per dataset and endpoint after standardisation are presented 
in Table 1. The corresponding numbers before standardisation can be found in the Additional file 1: Table S1.

\section{Compound encoding}

Converting molecules into numerical data was performed using the signature molecular descriptor [32, 33], using the program CPSign [34] version 0.7.14. The signature descriptor has been used extensively in previous QSAR studies [35-37]. In brief, the signature molecular descriptor enumerates all fragments of a molecule using a specified number of atomic bonds, often referred to as height, here using height 1 to 3 (e.g., height 1 creates fragments containing a center atom and all its one-bond connected atoms). This descriptor is often extremely sparse as there is a large number of fragments in a dataset and each molecule contains only a small set of these fragments. Herein, the count of each fragment was used; it is also possible to use a bit-type vector, where $0 / 1$ indicates whether the fragment is present or not. The composition of the training set and hence the number of descriptors is different per endpoint. On average 36,721 ( $\pm 2363 \mathrm{std}$ ) fragments were defined per endpoint in the Tox21Train set, whereas the signatures for Tox 21 Test and Tox21 ccore are based on the fragments in Tox21Train.

\section{Modelling}

\section{Conformal prediction}

Conformal prediction (CP) is a statistical framework, which provides means for confidence estimation $[15,38]$. The baseline conformal predictor is the computationally efficient inductive conformal predictor (ICP) [23] (indicated in purple in Fig. 1a). An ICP operates on the output from an underlying model. To allow calibration of the outputs, the training set is divided into a proper training set and a calibration set. An underlying model, most often a machine learning model, is fitted on the proper training set, predictions are made for both the test and the calibration set compounds, and transformed into socalled nonconformity scores. In a binary Mondrian setting $[38,39]$, for each test compound two p-values are calculated, one per class, by comparing the outcome of each instance with the outcomes of the corresponding calibration set compounds. Given the two p-values and a predefined significance level $\epsilon=1$ - confidence level, a prediction set is calculated. The prediction set contains all class labels, for which the p-value is larger than the significance level. For more information on conformal prediction, see Alvarsson et al. [40] and Norinder et al. [41].

\section{Aggregated conformal prediction methods}

To reduce the variance in efficiency of ICPs, multiple conformal predictors can typically be aggregated [42, 43] (see Fig. 1). In the commonly used aggregated conformal prediction (ACP) [43] aggregation method, the training set is randomly split $\mathrm{n}$ times into a proper training set and a calibration set, with which n ICPs are trained and calibrated (Fig. 1a). The p-values resulting from the different ICPs are then averaged. While the consolidation of multiple models stabilises the predictions, a uniform distribution of the p-values is not necessarily observed after their averaging [42].

The influence of ACPs on the calibration can be analysed by additionally incorporating the recently developed synergy conformal prediction (SCP) method (Fig. 1b) [44]. In the SCP, one fixed calibration set is

Table 1 Number of compounds (separated as actives and inactives) available per Tox21 dataset and endpoint after standardisation. The full names for the endpoints are adopted from Huang et al. [31]

\begin{tabular}{|c|c|c|c|c|c|c|}
\hline \multirow[t]{2}{*}{ Endpoint } & \multicolumn{2}{|c|}{ Tox21Train } & \multicolumn{2}{|c|}{ Tox21Test } & \multicolumn{2}{|c|}{ Tox21Score } \\
\hline & Actives & Inactives & Actives & Inactives & Actives & Inactives \\
\hline Aryl hydrocarbon receptor (NR_AhR) & 933 & 6687 & 29 & 236 & 71 & 506 \\
\hline Androgen receptor, full length (NR_AR) & 373 & 8370 & 3 & 282 & 11 & 549 \\
\hline Androgen receptor, ligand binding domain (NR_AR_LBD) & 295 & 7742 & 4 & 242 & 8 & 543 \\
\hline Aromatase (NR_Aromatase) & 338 & 6362 & 18 & 192 & 36 & 466 \\
\hline Estrogen receptor, full length (NR_ER) & 901 & 6290 & 27 & 231 & 49 & 441 \\
\hline Estrogen receptor, ligand binding domain (NR_ER_LBD) & 419 & 7763 & 10 & 270 & 20 & 548 \\
\hline Peroxisome proliferator-activated receptor gamma (NR_PPAR) & 204 & 7414 & 15 & 245 & 31 & 543 \\
\hline $\begin{array}{l}\text { Nuclear factor (erythroid-derived 2)-like 2/antioxidant responsive } \\
\text { element (SR_ARE) }\end{array}$ & 1032 & 5653 & 47 & 181 & 89 & 433 \\
\hline ATAD5 (SR_ATAD5) & 322 & 8179 & 25 & 240 & 36 & 554 \\
\hline Heat shock factor response element (SR_HSE) & 386 & 7233 & 10 & 250 & 19 & 558 \\
\hline Mitochondrial membrane potential (SR_MMP) & 1094 & 5719 & 38 & 195 & 56 & 457 \\
\hline p53 (SR_p53) & 515 & 7542 & 28 & 234 & 40 & 543 \\
\hline
\end{tabular}




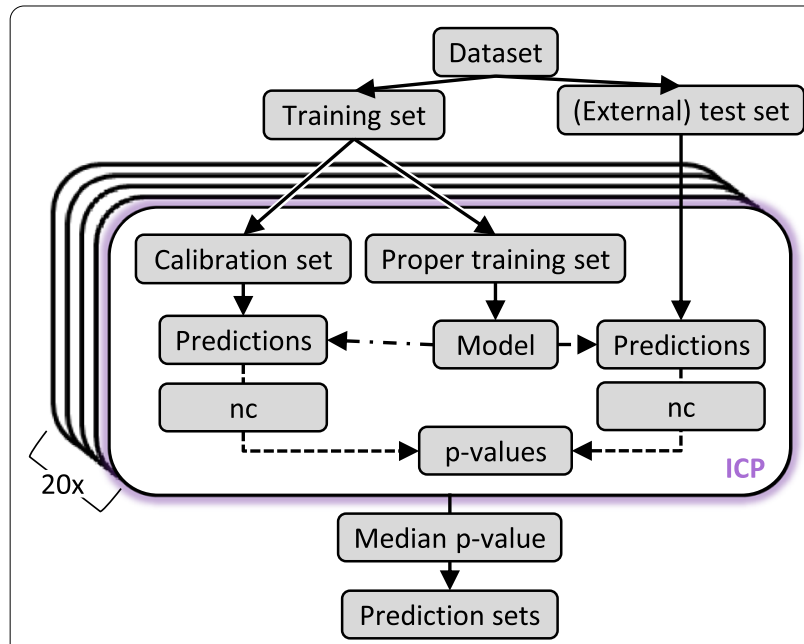

a Aggregated conformal predictor (ACP)

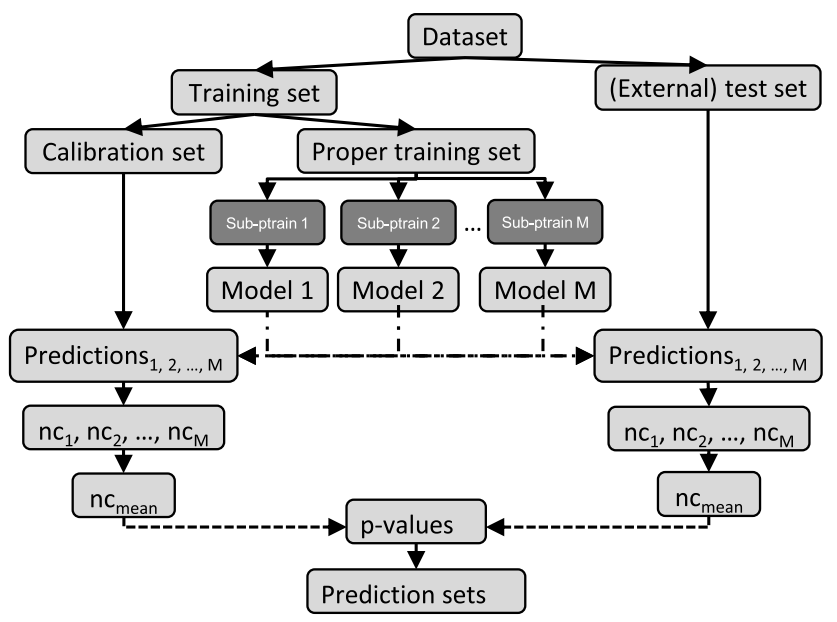

b Synergy conformal predictor (SCP)

Fig. 1 Inductive conformal predictor (ICP) and the aggregated conformal prediction methods used in this study. a Aggregated conformal prediction (ACP) and ICP (box with purple edge): The dataset is split into a training set and a test set. The training set is further split into a proper training set to train the model and a calibration set. The predictions made for the test set compounds are used to calculate nonconformity scores (nc) and compared to nonconformity scores in the calibration set to calculate $\mathrm{p}$-values and generate prediction sets. In ACP, multiple models are trained and calibrated with randomly selected proper training and calibration sets, and p-values from these are averaged. $\mathbf{b}$ Synergy conformal prediction (SCP): In order to ensure a uniform distribution of p-values, SCP averages the nonconformity scores instead. Multiple models are trained on (subsets of) the proper training set and with each model predictions are made for the test set and for a fixed calibration set

randomly selected, and the proper training set is split into $\mathrm{n}$ subsets to train multiple sub-models. Note that the analysis of other options to build an SCP, e.g. training several models using different $\mathrm{ML}$ algorithms on the same (sub)set, is out of scope for this work. The predictions made with every sub-model are aggregated before calculating the $\mathrm{p}$-values and prediction sets. A fixed calibration set reduces the number of available training compounds, but the needlessness of averaging p-values ensures a uniform distribution of the latter and hence leads to theoretically valid models [44].

\section{Model evaluation}

CP models are typically evaluated by their validity and efficiency [15]. Validity, for a given significance level, is defined as the ratio of prediction sets that contain the true label. The efficiency of a model is a way to measure the information content of the model, and we herein use the most widely used efficiency metric: ratio of single label sets at a given significance level. In binary CP, the possible prediction sets are $\{\emptyset\},\{0\},\{1\}$ and $\{0,1\}$, where only the $\{0\}$ and $\{1\}$ (i.e. single label sets) are informative, and 'empty' and 'both' sets are uninformative in a sense. Thus, the fraction of single label sets should be maximised for best efficiency.

\section{Model calibration}

When evaluating the predictive performance on a test set, deviations from the underlying assumption that all data come from the same distribution will lead to predictions that are invalid and hence the results might be misleading. In this work, we use calibration plots to identify deviations from acceptable levels of calibration, and also discuss potential mitigation strategies.

\section{Assessing model calibration}

In a conformal prediction setting, the observed error rate of predictions is theoretically proven to not be larger than the specified significance level. In return, any deviations between these values may indicate data drifts (or other causes for the deviations, such as a too small test set). The level of calibration can be visualised in a so-called calibration plot, where the observed error rate (y-axis) is plotted versus the significance level (desired error rate, x-axis). For valid (well-calibrated) models the values should lie on the diagonal line. Deviations from this behaviour signals deviations from perfect calibration. We also include efficiency in the plot, calculated as the fraction of single-class predictions. These plots, from hereon called calibration and efficiency plots (CEPs), were used in this work to assess the model calibration and efficiency (see Fig. 2). As a measure of the level of calibration, we 


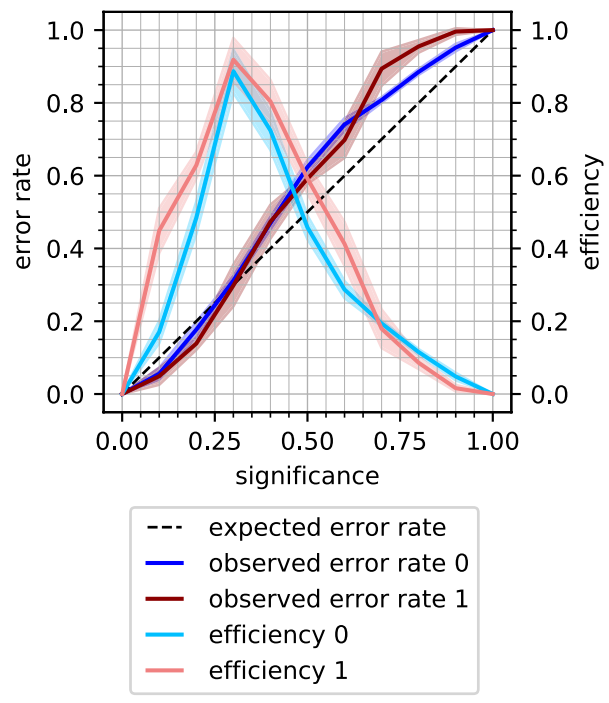

Fig. 2 Calibration and efficiency plot. The dark lines show the mean error rate for the active (dark red) and inactive (dark blue) compounds. For a well-calibrated model, the error rate ideally follows the dashed diagonal line. The light coloured lines illustrate the mean efficiencies expressed as ratio of single label sets for the active (light red) and inactive (light blue) compounds. The shaded areas indicate the respective standard deviations within the fivefold CV. Class 0: inactive compounds, class 1: active compounds

use the root-mean-square deviation (RMSD) between the specified significance and the observed error rate.

\section{Model update strategies}

In a setting where a model has been trained but new data on the same or a similar endpoint is made available, it is interesting to consider how the new data should be utilised in order to improve primarily the level of calibration but also the efficiency. We investigated two update strategies, see Fig. 3. The first strategy included updating the whole training set with new data followed by subsequent retraining of the model (see Fig. 3a). In the second strategy, the proper training set was kept and only the calibration set was exchanged with more recent data (see Fig. 3b).

\section{Study design}

In this work, six different $\mathrm{CP}$ experiments were explored as illustrated in Fig. 4 and Table 2. The first experiment consisted of a cross-validation (CV) using ACP on the Tox21Train dataset (1-internal_CV), the second comprised predictions with the CV-models from experiment 1 on the Tox 21Score dataset (2-pred_score). In the third experiment, the influence of ACP on the calibration was assessed by training an SCP model on Tox21Train and predicting Tox21Score (3-pred_score_SCP). Finally, in the last three experiments, the model update strategies to improve the calibration were evaluated (see Fig. 3). Thus, in experiment 4 the training set was updated (4-train update) and the model retrained, while in experiment 5 and 6 only the calibration set was updated (5-cal_update and 6-cal_update_2).

The individual experiments were conceptualised in a way that the proper training sets were consistent across all experiments (where applicable). A fivefold CV was implemented, not only for internal validation (1-internal_CV), but conserved for all experiments. Hence, the selected data per CV loop of a fivefold CV were retained for all trained models (i.e. in the 1-internal_CV, 4-train update and 3-pred_score_SCP experiments). Specifically, the indices of the training compounds were saved, so that the same training sets could be used for the subsequent experiments. This ensures that the results from the different experiments can be directly compared. For the ACP model, 20 aggregated ICPs were used with $30 \%$ (of the training set) set aside as a calibration set and $70 \%$ as a

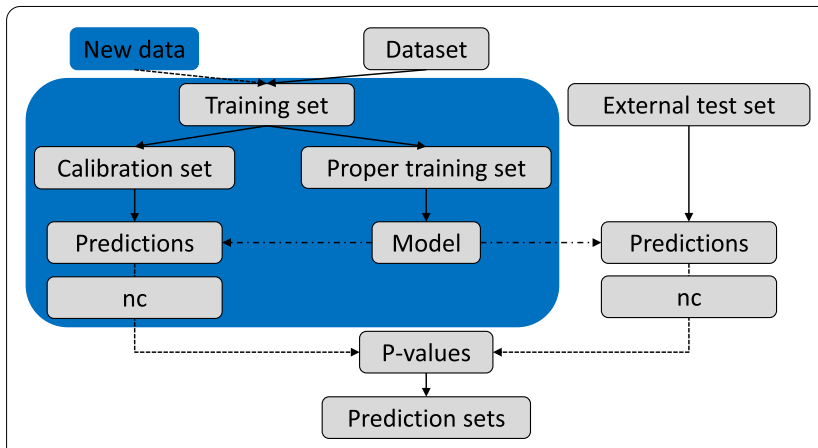

a Update training set

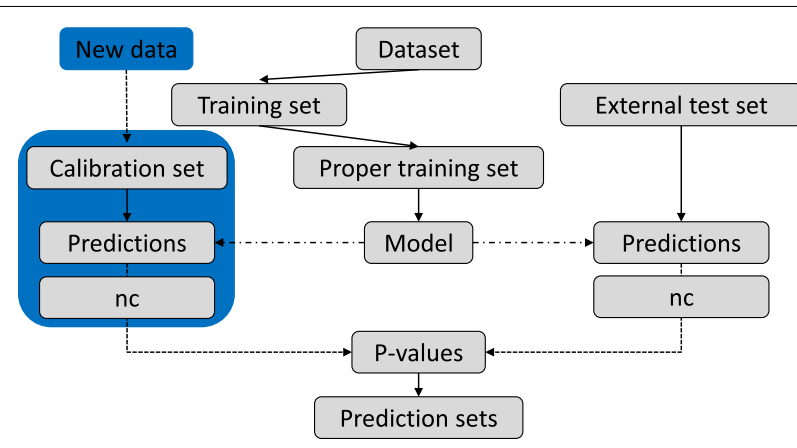

b Exchange calibration set

Fig. 3 Model update strategies analysed to improve calibration. a Update training set:The whole training set is updated with new data. This involves retraining a new model. b Exchange calibration set: Only the calibration set is updated with new data. Models can hereby be re-calibrated without training a new model 


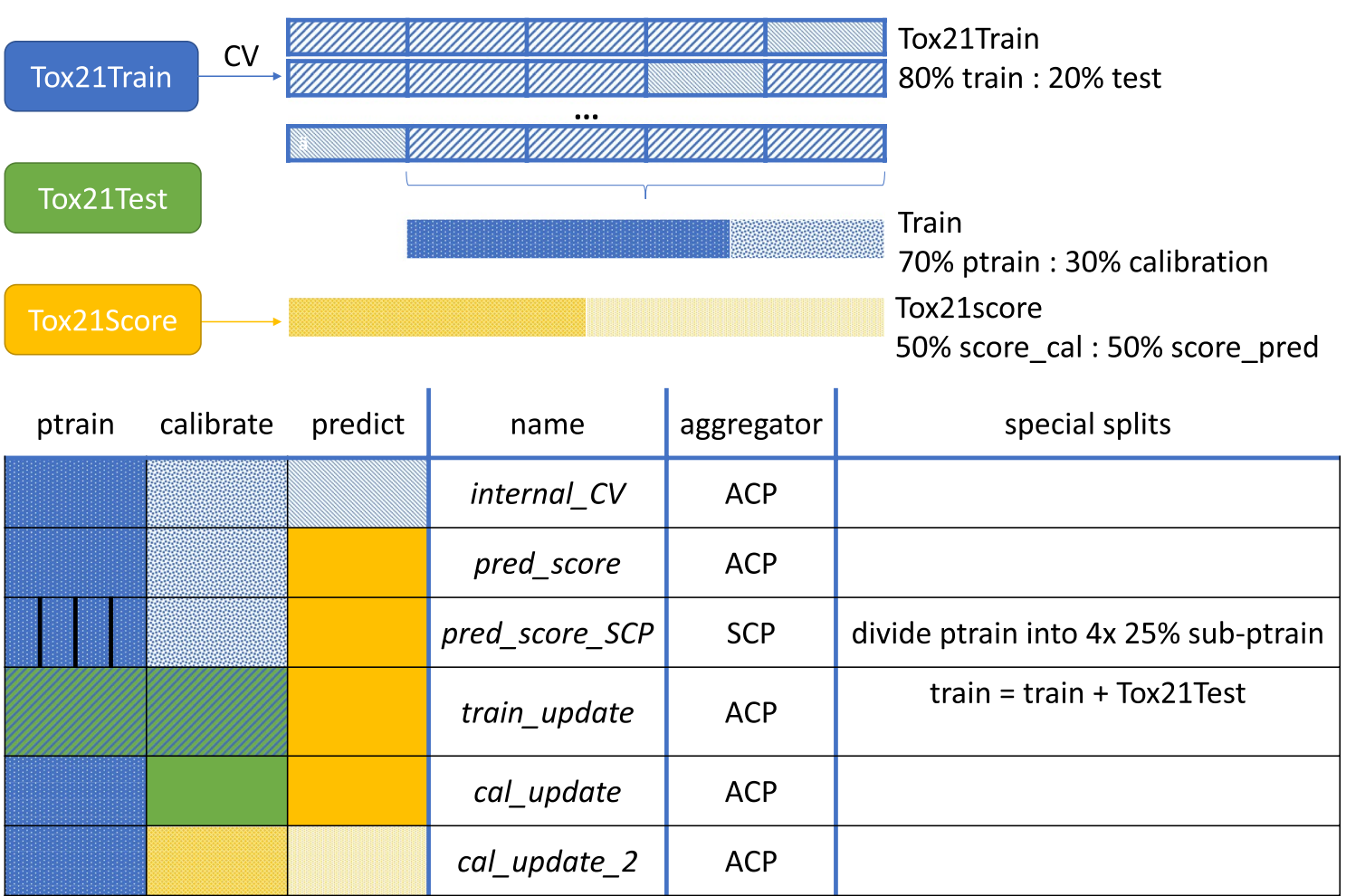

Fig. 4 Overview of the experiments discussed in this work. Top: Splitting of Tox21 data into (proper) training, calibration and test set. Bottom: Data for training, calibration, and prediction as well as aggregator used in the specific experiments.

Table 2 Overview of the experiments discussed in this work. Note that all splits were performed randomly stratified

\begin{tabular}{|c|c|c|}
\hline Nr. & Name & Explanation \\
\hline 1 & internal_CV & $\begin{array}{l}\text { A fivefold CV, training one ACP per fold, is performed on the Tox } 21 \text { Train dataset and internally evaluated on the respective hold } \\
\text { out data. }\end{array}$ \\
\hline 2 & pred_score & Using the CV-models trained within the above described CV, the Tox21Score data are predicted. \\
\hline 3 & pred_score_SCP & $\begin{array}{l}\text { The same CV splits are applied as described above. The training set is then split into a fixed calibration set and four proportion- } \\
\text { ate sub-proper training sets. For each of the four corresponding sub-proper training sets, an ML model is trained. Predictions } \\
\text { are made for Tox } 21 \text { Score (and the calibration set compounds) with every model; the four nonconformity scores (ncs) are } \\
\text { averaged before calculating the p-values. }\end{array}$ \\
\hline 4 & train_update & $\begin{array}{l}\text { The training set from the CV is combined with the Tox } 21 \text { Test set. This updated training set is then split into proper training and } \\
\text { calibration set to train new ACP models for the CV set-up. Tox21Score data are predicted with the new models. }\end{array}$ \\
\hline 5 & cal_update & The CV-models from experiment 1 are used, but the calibration is updated with the Tox21Test data to predict Tox21Score. \\
\hline 6 & cal_update_2 & $\begin{array}{l}\text { The CV-models from experiment } 1 \text { are used, but the calibration is updated with } 50 \% \text { of Tox } 21 \text { Score data. The other } 50 \% \text { of } \\
\text { Tox } 21 \text { Score are predicted. In every fold of the CV, Tox } 21 \text { Score is split in two equal subsets. }\end{array}$ \\
\hline
\end{tabular}

proper training set. For the 3-pred_score_SCP experiment (using SCP, see Fig. 1a), the training set was split into a fixed $30 \%$ calibration set and the proper training set divided into four equally sized partitions. For the 4-train update experiment, the training set was first updated with the Tox21Test dataset and then split into calibration and proper training set using the above described ratios. For the two experiments updating the calibration set, the same trained CV-model from 1-internal_CV was calibrated with only the Tox 21 Test dataset (5-cal_update) and in the last experiment (6-cal_update_2) replacing the calibration examples with 50\% randomly stratified split Tox21Score data.

SVM models were trained using the Scikit-learn Python library [45] version 0.23 .2 with an RFB kernel, $\mathrm{C}=50, \gamma=0.002$ ) [37]. For conformal prediction, the nonconformist Python library [46] was used with margin error function, Mondrian condition [38, 39] version 2.1.0. 
For ACP, p-values were aggregated by median (see [42]), for SCP the nonconformity scores were averaged before calculating $\mathrm{p}$-values.

\section{Code and data availability}

A GitHub repository associated with this work is available at https://github.com/volkamerlab/cptox21_manus cript_SI. It contains the signature fingerprints for all pre-processed datasets as well as example code to demonstrate how the different ACP experiments were performed. The repository also provides the result files containing the respective measures for all experiments, from which the CEPs and boxplots can be generated. The SCP code is available from the original SCP repository by Gauraha et al. [44, 47].

\section{Results and discussion}

The aim of this study was to assess the level of calibration between the initial release of the Tox21Train data and the subsequently released Tox 21 Score data using conformal prediction (experiments 1-3). In follow-up experiments, we also investigated two model update strategies for incorporating the Tox21Test data (experiments 4-6). An overview of the error rates and efficiencies at significance level 0.2 for all experiments is provided in the Additional file 1: Table S2.

\section{Experiment 1: Cross-validation on the Tox21Train datasets}

Before applying a model to external data, it needs to be validated by ensuring that the model is internally well calibrated. Hence, in a first experiment (1-internal_CV), models were built in a fivefold CV scenario on the Tox21Train datasets. The models for the 12 Tox 21 endpoints were internally valid with a mean error rate of 0.17 ( \pm $0.01 \mathrm{std})$ at significance level 0.2 , as well as a high mean efficiency of 0.77 ( $\pm 0.13 \mathrm{std})$.

The error rates and efficiencies over all significance levels (mean and std of the five CV folds per model) are illustrated in CEPs (Fig. 5a) for three example endpoints (namely SR ARE, NR_Aromatase and NR_AR; the

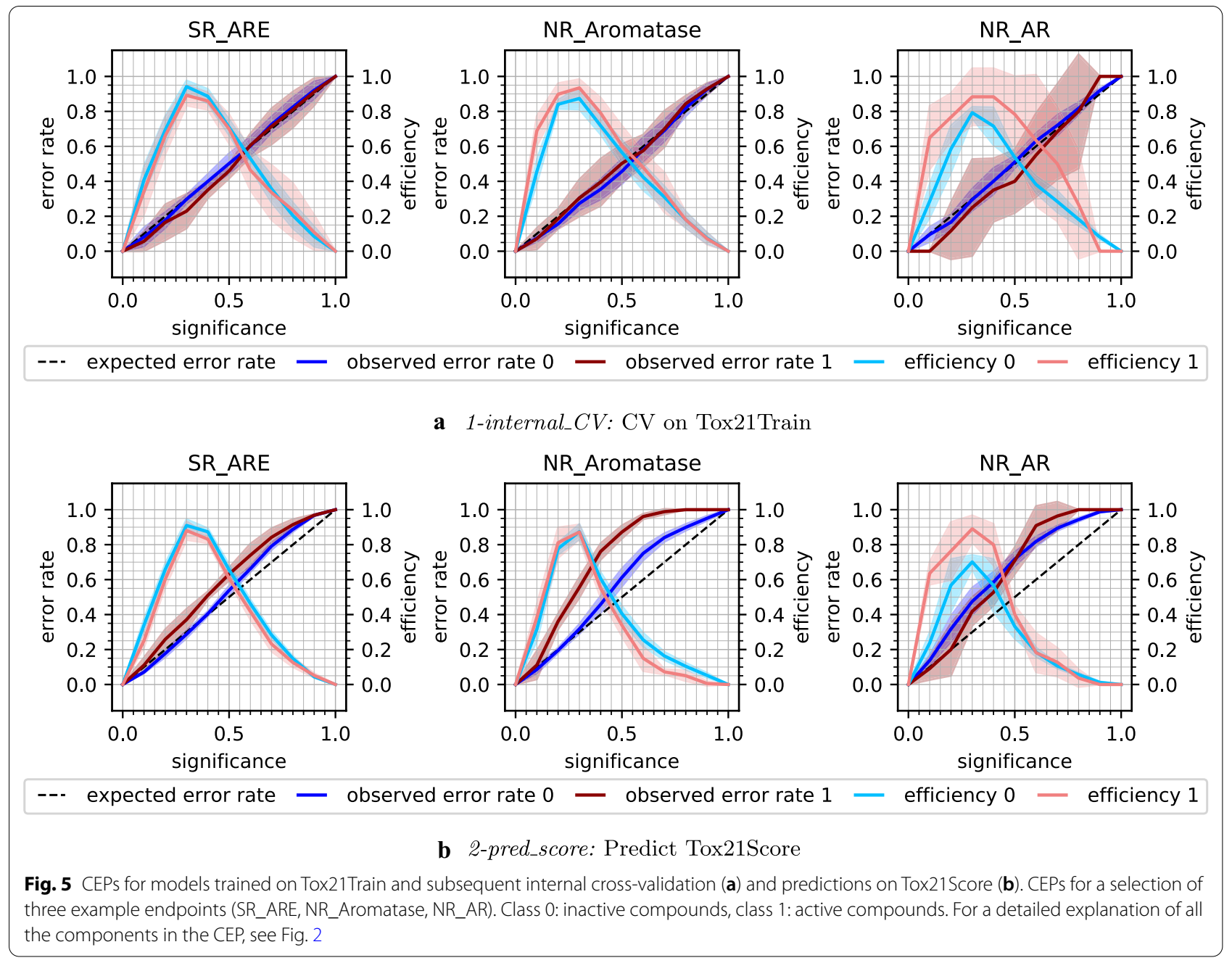


remaining CEPs are shown in the Additional file 1: Figure S1). While the models are overall well calibrated, i.e. the observed error rates follow the diagonal line in the CEPs, and the standard deviations between the individual runs are low, there are a few outliers. The high variance (see shaded areas in the CEPs) for the active compounds and the low efficiency for NR_AR reflect the observations in the Tox21 data challenge that NR_AR was one of the most difficult targets to model and has, with 387 active and 9201 inactive compounds, the lowest active compound rate after NR_PPAR $\gamma$ and NR_AR_LBD [31]. The well-calibrated models were ready to be applied to external data which stem from the same distribution as the training data.

\section{Experiment 2: Model performance on the Tox21Score datasets}

To investigate how well the CP models from the crossvalidation perform on an external dataset, predictions were made for the Tox 21 Score data (2-pred_score). A mean error rate at significance level 0.2 of $0.31( \pm 0.12$ std) was achieved. The efficiency dropped only slightly to 0.72 ( $\pm 0.14 \mathrm{std})$. The deviations from the diagonal line in the CEPs (Fig. 5b, Additional file 1: Figure S2) for most of the endpoints indicate that the calibration of the models was poor when predicting Tox21Score.

Note that predictions were also made for the Tox21Test compounds (shown in the Additional file 1: Figure S3 only, referred to as pred_test). This set-up was similar to the intermediate setting in the Tox 21 challenge, where predictions on Tox21Test were decisive for the leaderboard. The mean error rate at significance level 0.2 over all endpoints was higher than expected $(0.26 \pm 0.11 \mathrm{std})$. So, the models were not well-calibrated for predictions on Tox21Test. The mean efficiency was 0.70 ( $\pm 0.15 \mathrm{std})$, i.e. similar to 2-pred_score results. The poor calibration for the predictions on both (external) datasets is an indication that the Tox21Score and the Tox21Test data might come from a different distribution than the Tox21Train data.

\section{Experiment 3: Influence of aggregation method on the calibration}

Reasons for poor calibration can be the difference between the distribution of two datasets, but also the data set size (discussed later) or the aggregation strategy for the conformal predictor (here ACP). From a theoretical perspective, the use of ACP can affect the calibration, as ACPs have not been proven to be always valid [42]. In $\mathrm{ACP}$, the $\mathrm{p}$-values from all ICPs are aggregated, which in theory could result in a non-uniform distribution. To rule out that the use of $\mathrm{ACP}$ is the (main) reason for the poor calibration, the recently developed SCP aggregation method was applied. In the SCP framework (see Fig. 1b), nonconformity scores are averaged before calculating the p-values, which are the basis for the calibration. This aggregation method has been shown to be theoretically valid [44].

Applying SCP improved the calibration on the Tox21Score dataset (3-pred_score_SCP), the mean error rate decreased to 0.27 ( $\pm 0.12 \mathrm{std})$ and the mean efficiency at significance level 0.2 was 0.73 ( \pm 0.13 std). The error rates and efficiencies over all significance levels are shown in the CEPs in Fig. 6 for the SR_ARE, NR_Aromatase, and NR_AR endpoints and in the Additional file 1: Figure S4 for all 12 endpoints. It is especially noticeable that the calibration curves in the CEPs became less sigmoidal for many endpoints-such sigmoidal curves have typically been observed for ACPs [42, 44]. The sigmoidal shape is unfavourable from a theoretical perspective as it means

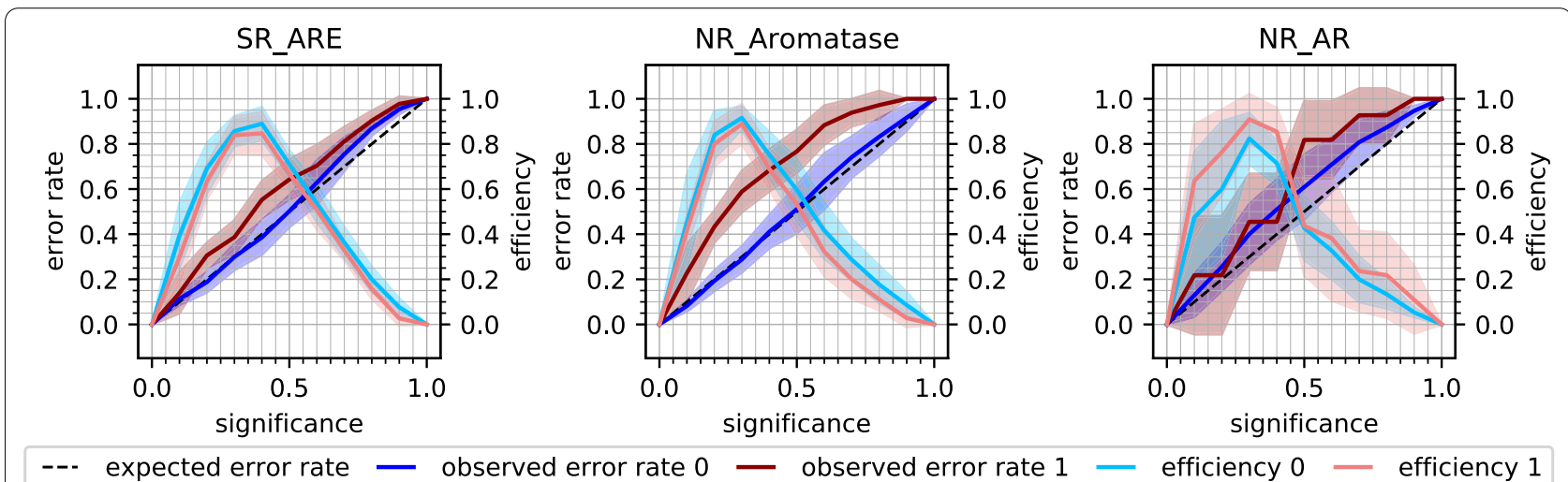

Fig. 6 Results from experiment 3-pred_score_scp: SCP models were trained on Tox21Train and predictions made for Tox21Score. CEPs are shown for a selection of three example endpoints (SR_ARE, NR_Aromatase, NR_AR). Class 0: inactive compounds, class 1: active compounds. For a detailed explanation of all the components in the CEP, see Fig. 2 
that the model is poorly calibrated at low and high significance levels, but may be less problematic in an application context since the error rate is typically over-conservative at lower (i.e. relevant) significance levels. One drawback of SCP is the fixed calibration set, which means that part of the training set information is never used for training. Together with the smaller proper training set partitions, this can lead to less efficient predictions. This can be seen in the relatively large standard deviations of the error and efficiency rates in the CEPs (Fig. 6 and Additional file 1: Figure S4). For this reason, and since ACP is commonly used in literature, which makes the outcomes more comparable with work by other scientists, ACP was used for the subsequent experiments.

Summarising the results from experiment $1-3$, it was concluded that the Tox21Test and Tox21Score data may originate from slightly different distributions than the Tox21Train data. This could be explained by knowing that the three Tox 21 datasets were created (screening of compounds) at different stages. For the Tox21Train set, the actual "Tox21 10K dataset" [31] was used, for which data had been available at the start of the challenge. The Tox21Test dataset is part of the LOPAC ${ }^{1280}$ (Library of Pharmacologically Active Compounds) dataset, which was used to validate the Tox21 assays [31, 48]. The Tox21Score data were separately provided by the EPA and only screened during the challenge [31]. So-called data or assay drifts typically occur over time or when moving towards a different chemical space [49].

\section{Experiment 4: Effects on calibration by updating the training set}

When the model is not well calibrated for the predictive task and newer data are available, one would intuitively combine these additional data (i.e. Tox21Test) with the previous training data (i.e. from 1-internal_CV), train a new model, and use it to predict Tox21Score (4-train update). Following this strategy, the mean error rate over the 12 endpoints dropped to 0.23 ( $\pm 0.06 \mathrm{std}$ ) compared to the predictions with the model built on the Tox21Train data (2-pred_score, $0.31 \pm 0.12 \mathrm{std})$. The mean efficiency at significance level $0.2(0.71 \pm 0.15 \mathrm{std})$ was in a similar range as with the original training set $(0.72 \pm 0.14$ std). Thus, the updating of the training set and retraining the model led to a small improvement in calibration (see CEPs in Additional file 1: Figure S5). One reason why we observed only a minor improvement of the calibration could be the sizes of the two datasets. The update set (254 \pm 22 compounds) is small compared to the original training set (7647 \pm 692 compounds) and has thus a lower influence on the new model. Furthermore, this strategy involves additional computational resources and the data of the previous model needs to be available for retraining.

\section{Effects on calibration by updating the calibration set Experiment 5: Replace the calibration set with observations from Tox21Test}

An alternative to updating the whole training set is to replace only the calibration set with the more recent data. This comes with the additional advantage that the calibration set can be renewed even if the training data are unavailable.

Updating the calibration set did result in a lower mean error rate of 0.21 ( $\pm 0.05 \mathrm{std})$ for the predictions on Tox21Score (5-cal_update). The mean efficiency at significance level 0.2 dropped to 0.51 ( $\pm 0.18 \mathrm{std})$. The loss in efficiency at low significance levels can be observed in the CEPs (Fig. 7a and Additional file 1: Figure S6), where the peak in efficiency is shifted towards higher significance levels. In the same CEPs, the improved calibration can be seen in the lower error rates. For six endpoints, when considering inactive compounds, or 11 endpoints, with regard to active compounds, even overconservative validity, i.e. a lower than expected error rate was achieved.

\section{Experiment 6: Exchange the calibration set with half of Tox21Score}

The chronological order of how the experimental data were produced is given by the Tox 21 challenge organisers [31]. However, it is not clear if the compounds contained in Tox21Score (and Tox21Test) were really developed later than those in Tox21Train. For a 'perfect' calibration, it is required that the calibration and the test set stem from the same distribution. To simulate this, a second updating experiment, i.e. 6-cal_update_2, was implemented. While still using the same proper training set as for the former experiments, the updated calibration set was created from Tox $21 S c o r e$. In every of the five (original) CV folds, $50 \%$ of Tox $21 S$ core was (randomly stratified) selected to constitute the calibration set while the other $50 \%$ of Tox 21 Score was used as test set. With this set-up, calibration and test set originate from the same distribution. This was also reflected in the mean error rate of 0.18 ( $\pm 0.01 \mathrm{std})$ at significance level 0.2 , which was in a similar range as for the 1-internal_ $C V$ with the original calibration set $(0.17 \pm 0.01 \mathrm{std})$. Similar to the previous updating experiment 5-cal_update, the efficiency decreased to 0.50 ( $\pm 0.17 \mathrm{std})$ at significance level 0.2 . Note that also the size of the calibration set was similar to the former 5-cal_update experiment, as the Tox21 Score set contains roughly twice as many compounds $(551 \pm 35)$ as Tox21Test $(254 \pm 22)$. On the other hand, by using half of Tox 21 Score for calibration, only the other half of the compounds was available for use as test set. This could lead to higher variations, e.g. in the error rate, especially for datasets with few test compounds. Such an example is shown for the NR_AR endpoint, for which 


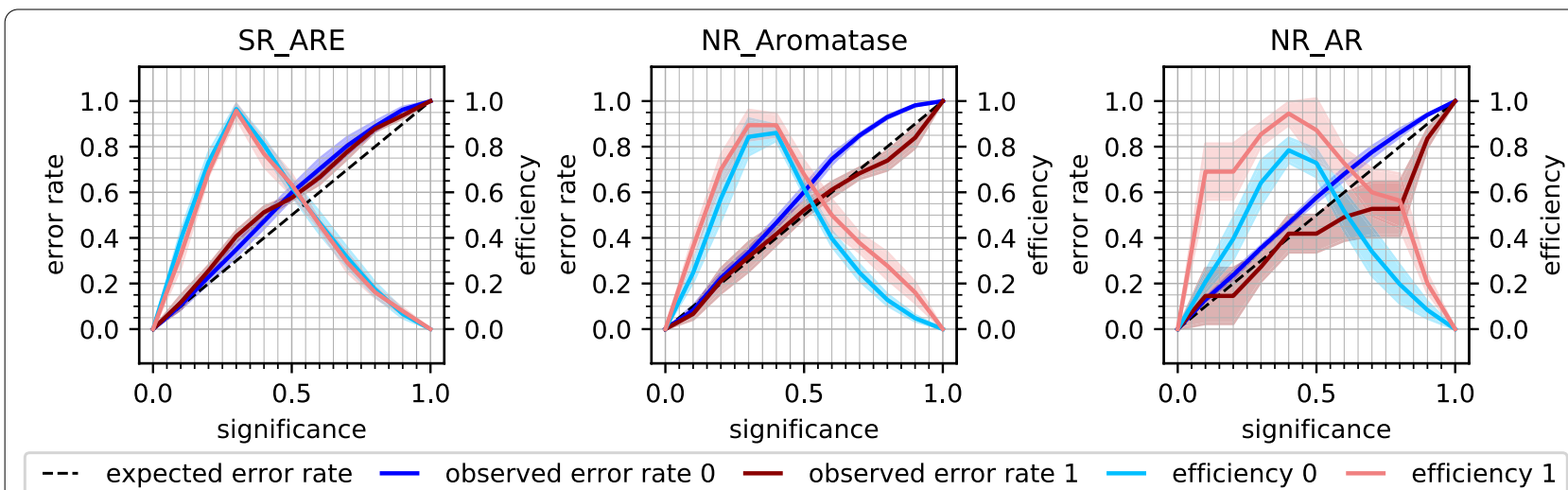

a 5-cal_update: Exchange calibration set with Tox21Test
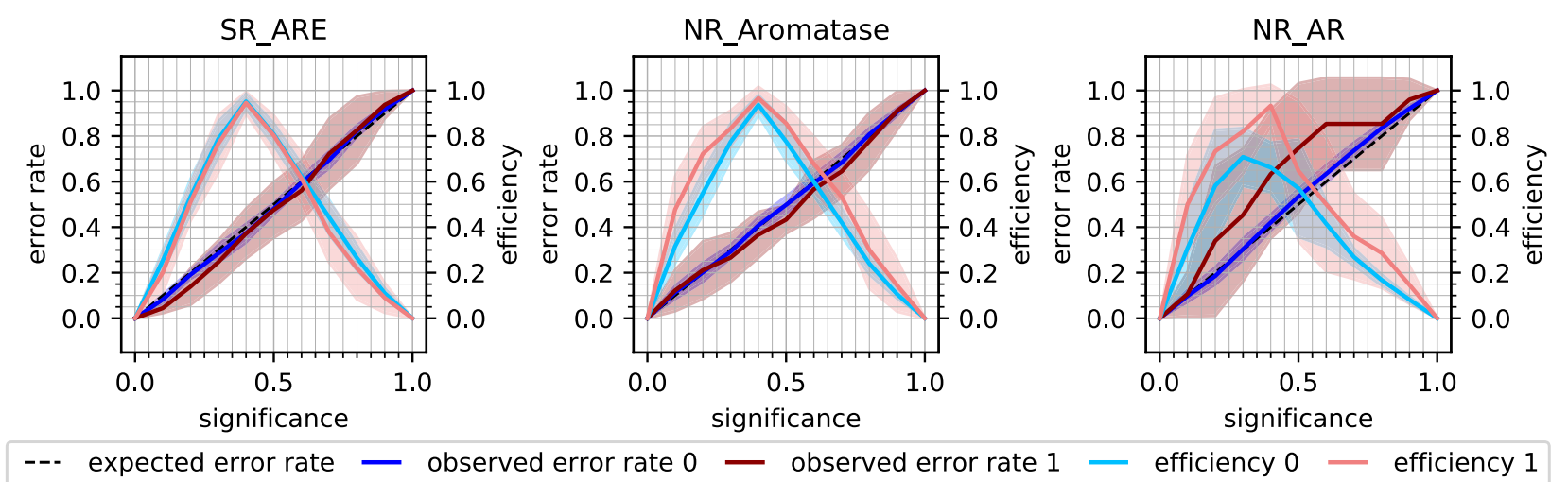

b 6-cal_update_2: Exchange calibration set with half of Tox21Score

Fig. 7 Updating the calibration set with more recent data from Tox21Test (a) or Tox21Score (b). CEPs for a selection of three example endpoints (SR_ARE, NR_Aromatase, NR_AR). Class 0: inactive compounds, class 1: active compounds. For a detailed explanation of all the components in the CEP, see Fig. 2

Tox21Score only contains 11 actives. The standard deviation (shaded area) for the error rate and efficiency of the active compounds (red) increased compared to the 5-cal_ update experiment (Fig. 7). For the other two example endpoints in Fig. 7b (SR_ARE and NR_Aromatase), the calibration improved considerably. Summarising, the CEPs in the Additional file 1: Figure S7 illustrate how the calibration improved after exchanging the calibration set with data from the same distribution as the test set, but also how the efficiency dropped compared to the 4-train_update strategy (Additional file 1: Figure S5). The decrease in efficiency in the 'cal_update' experiments is undesired but can be an acceptable trade-off in cases where validity could be restored. However, it has to be noted that the 6-cal_update_2 scenario is not often practically applicable as the updated calibration data needs to be available before making predictions.

Ultimately, updating the calibration set has no impact on the applicability domain of the underlying model.
Improved calibration level and lower efficiency rather indicate that more compounds outside the applicability domain might be detected and classified as 'both' prediction sets. Thus, applying the 5-cal_update over the 4-train_update strategy is mainly promising in a situation as described in this work where the number of available new compounds is limited.

\section{Quantification of the calibration for all experiments}

The error rates (discussed above) depend on the desired significance level. In the calibration plot, the error rates are plotted over a range of significance levels. However, if the model will only be applied at a certain significance level, obtaining a good level of calibration at that significance level might be enough. But, if the calibration of the model is assessed from a theoretical perspective, all significance levels must be considered. This was illustrated for the individual experiments with the help of CEPs as 
discussed above. To have a comparable metric, the rootmean-square deviation (RMSD) over all significance levels (step-width 0.1) was calculated.

Boxplots illustrating the RMSDs between observed and expected error rates over all endpoints are available in Fig. $8 \mathrm{a}$ for the active compounds and Fig. $8 \mathrm{~b}$ for the inactive compounds, and show how the error rate deviations behave between the individual experiments. The mean RMSD values (overall, actives and inactives) for all experiments are provided in the Additional file 1: Table S3.

Clearly, the RMSD for the actives and inactives is low in the internal CV with Tox21Train (1-internal_CV) for most of the endpoints (mean overall RMSD: 0.022), while the deviations increased for the predictions on Tox21Score (2-pred_score, mean overall RMSD: 0.150). When using the SCP aggregation method (3-pred_score_ $S C P)$, the RMSD decreased for eight endpoints, albeit, only by a small amount (Fig. 8, mean overall RMSD: 0.121). Updating the training set (4-train_update, using ACP) led only to a small improvement of the mean RMSD of the active compounds (mean RMSD, actives: 0.135 , Fig. 8a), while the improvement was more distinct for the inactive compounds (mean RMSD, inactives: 0.089 , see Fig. $8 \mathrm{~b}$ ). When exchanging the calibration set with Tox21Test (5-cal_update), the RMSD decreased for 11 endpoints (except for SR_ARE, for which the calibration was already very good (overall RMSD SR_ARE, 1-pred_score: 0.055 ) with the original calibration set). The mean overall RMSD (0.054) was, however, still not at the same level as for 1-internal_CV. This can be attributed to overconservative validity, especially for the active compounds (see Additional file 1: Figure S6) which led to an increased RMSD for several endpoints. The overconservative validity almost disappeared when the calibration set was exchanged with data which are inherently exchangeable with the test set (6-cal_update_2). The mean RMSD (0.018) value of the inactive compounds is at a similar level as for the internal CV on Tox21Train (1-internal $C V$ ) as shown in Fig. 8b. The RMSD values of the active compounds vary more between the different endpoints. This may be explained by the small number of active compounds available in the calibration and test sets for some endpoints. To summarise, the $\mathrm{CP}$ models trained on Tox21Train were internally well calibrated (1-inter$n a l_{-} C V$ ) but showed poorer calibration for the prediction of Tox21Score (2-pred_score). Applying SCP (3-pred score_SCP) or updating the training set with Tox21Test (4-train_update) did not improve the calibration to the same extent as when exchanging the calibration set only (5-cal_update, 6-cal_update_2).

\section{Impact of data size on the calibration}

Importantly, the proofs on $\mathrm{CP}$ validity are made assuming an asymptotic number of test examples (i.e. requiring an infinite number of test examples) [24]. Hence, the poor calibration is not necessarily only due to exchangeability issues (or the use of ACP, for which there are no validity guarantees). The calibration could also be affected by the

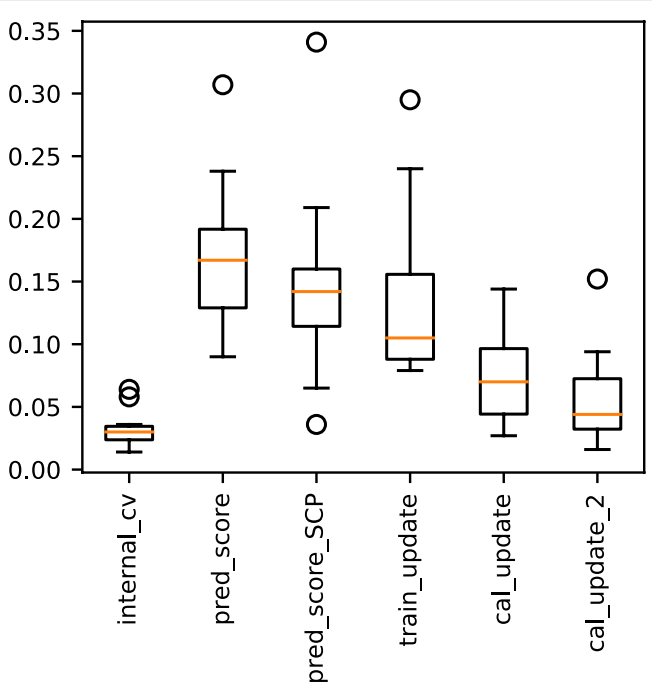

a RMSD actives

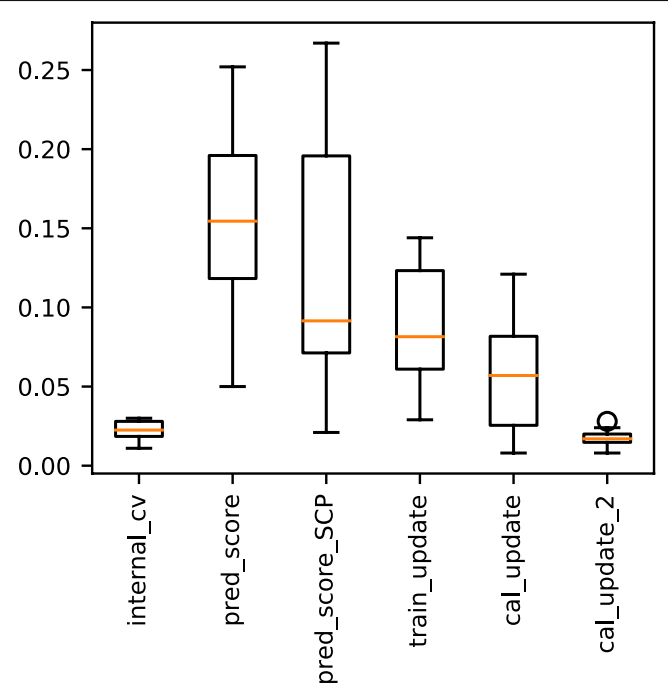

b RMSD inactives

Fig. 8 Box plots for the root-mean-square deviation (RMSD) between the expected and observed error rates for all 12 Tox21 endpoints compared amongst the different experiments are shown. On the left results for the active compounds (a), on the right for the inactive compounds (b) are plotted. Note that the $y$-axis ranges differ 
statistical variation due to finite test sets in all computational experiments. In the broadest sense, also the overconservative validity could be due to the finite number of test examples.

Looking at the outliers in the RMSD (Fig. 8), they mainly arise from endpoints NR_AR_LBD, NR_AR and SR_ATAD5, which are, besides NR_PPAR $\gamma$, the endpoints with the smallest overall number of actives (in all three Tox21 datasets combined). For the NR_AR and NR_AR_LBD datasets, the predictive performance (both in validity and efficiency) is expected to be less good for the active compounds, as the number of available active compounds is very small (i.e. 3 and 4 in Tox 21 Test and 11 and 8 in Tox21Score, respectively). If we have only eight compounds in the calibration set, this means that only nine different p-values can be obtained for a new active compound. This low resolution obviously makes it impossible to obtain perfect calibration. Since it is difficult to define a minimum required number of actives, and since the resolution for the p-values of the inactive compounds is much higher, results for all endpoints were included in the evaluation. The calibration might generally improve if the experiments were repeated on larger and/or more balanced datasets.

Although, the composition of the three Tox 21 datasets may not conform with all model assumptions, this may more closely resemble many real-life scenarios where data is generated at different time points and older data is often used to predict new outcomes. All the more, it is therefore important to have strategies to improve the calibration and thus the application of CP models on new data.

\section{Conclusions}

In this work, the potential of $\mathrm{CP}$ to diagnose data drifts in toxicity datasets was investigated on the Tox 21 data. Deviations between observed and expected error rates was monitored using calibration plots and quantified using the RMSD from the expected calibration level. Poor calibration was observed for models trained on Tox21 Train and predictions made on Tox21Score, indicating the presence of drifts between the two datasets. The distribution of the data may not be the only reason for error rate deviations in the calibration plot. In additional experiments using the newly introduced SCP framework, it was ruled out for 10 endpoints that the employed $\mathrm{CP}$ aggregation method (ACP) has a major impact. A second influencing factor on the calibration can be the small data set size. It was discussed that the calibration may be improved to some extent by having larger datasets, especially containing more active compounds, for model training, calibration and testing. Overall, it was concluded that the three Tox 21 datasets likely do not originate from the same distribution and may be challenging for ML methods. Nonetheless, these datasets do reflect outcomes that may occur in experimental screening scenarios.

Two different model update strategies using the intermediate Tox21Test data were investigated with the aim to improve the poor calibration. The calibration of predictions on Tox 21 Score could be slightly enhanced by updating the training set with more recent data (Tox21Test) and retraining the models - the more natural behaviour if new data has been obtained. However, exchanging only the calibration set with newer data (Tox21Test) led to a slightly smaller error rate, albeit often with a reduction in efficiency. As an additional advantage of the 5-cal_update strategy, retraining of the model is not required.

\section{Abbreviations}

ML: Machine learning; SVM: Support vector machine; EP: Endpoint; NR_AhR: Aryl hydrocarbon receptor; NR_AR: Androgen receptor, full length; NR_ER: Estrogen receptor, full length; NR_AR_LBD: Androgen receptor, ligand-binding domain; NR_ER_LBD: Estrogen receptor, ligand-binding domain; NR_PPAR_ $\gamma$ :Peroxisome proliferator-activated receptor gamma; SR_ARE: Nuclear factor (erythroid-derived 2)-like 2/antioxidant responsive element; SR_HSE: Heat shock factor response element; SR_MMP: Mitochondrial membrane potential; SR_ATAD5: ATAD5; NR_Aromatase: Aromatase; SR_p53: p53; CP: Conformal prediction; ICP: Inductive conformal predictor; ACP: Aggregated conformal prediction; SCP: Synergy conformal prediction; nc: Nonconformity score; RMSD: Root-mean-square deviation; Tox21Train: Training data for the Tox21 Data Challenge; Tox21Test: Test data for the Tox21 Data Challenge; Tox21Score: Final scoring data for the Tox21 Data Challenge; CEP: Calibration and efficiency plot; CV: Cross-validation; AD: Applicability domain.

\section{Supplementary Information}

The online version contains supplementary material available at https://doi. org/10.1186/s13321-021-00511-5.

Additional file 1: Table S1. Number of compounds available per Tox21 dataset and endpoint before standardisation. Table S2. Mean \pm standard deviation values over all twelve endpoints for observed error rate and efficiency at SL 0.2 for all experiments. Figure S1. 1-internal_CV: ACP models were trained and calibrated on Tox21Train and internally validated. Figure S2. 2-pred_score: ACP models were trained and calibrated on Tox21 Train and predictions were made for Tox21Score. Figure S3. pred test: ACP models were trained and calibrated on Tox21Train and predictions were made for Tox21Test. Figure S4. 3-pred_score_SCP: SCP models were trained on Tox21Train and predictions made for Tox21Score. Figure S5. 4-train_update: The training set from Tox21Train was updated with Tox21Test. Figure S6. 5-cal_update: ACP models were trained on Tox21Train and calibrated on Tox21Test. Figure S7.6-cal_update_2: ACP models were trained on Tox21Train and calibrated on 50\% of Tox21Score. Table S3. Mean RMSD values over all 12 endpoints, calculated for all compounds, as well as for active and inactive compounds, separately.

\section{Acknowledgements}

Computational resources were provided by SNIC-UPPMAX under project [SNIC 2019/8-149]. AM and AV would additionally like to thank the HPC Service of ZEDAT, Freie Universität Berlin, for computing time.

\section{Authors' contributions}

AM conducted the study under close supervision of UN, OS and $A \mathrm{~V}$, and in intense collaboration with all other project partners: FS, SAMS and NG. SAMS supported the signature generation with CPSign and NG provided support 
with the SCP framework. AM, FS, UN, OS and AV designed the study. AM wrote the first draft of the manuscript and all authors contributed actively to the text. All authors revised the final manuscript. All authors read and approved the final manuscript.

\section{Funding}

Open Access funding enabled and organised by Projekt DEAL. AM acknowledges support from FUBright Mobility Allowances and the HaVo-Stiftung. AV thanks BMBF (grant 031A262C) for funding. ARUK UCL DDI is funded by Alzheimer's Research UK (Grant No. 560832). OS is supported by Swedish FORMAS (Grant 2018-00924) and Swedish Research Council (Grants 2020-03731 and 2020-01865). NG and OS were supported by Swedish Foundation for Strategic Research (Grant BD150008).

\section{Availability of data and materials}

A GitHub repository with supplementary information is available under https://github.com/volkamerlab/cptox21_manuscript_SI. In the repository, the signature fingerprints for all pre-processed datasets are provided, as well as the output evaluation files from all experiments, which contain the underlying data for the CEPs and boxplots. The repository, also contains example code to demonstrate how the different ACP experiments were performed. For the SCP code, the reader is referred to the original SCP repo by Gauraha $[44,47]$.

\section{Declarations}

\section{Competing interests}

OS declares ownership of Aros Bio AB, a company developing the CPSign software. SAMS declares contributions to the CPSign codebase.

\section{Author details}

'In Silico Toxicology and Structural Bioinformatics, Institute of Physiology, Charité Universitätsmedizin, Berlin, Germany. ${ }^{2}$ Alzheimer's Research UK UCL Drug Discovery Institute, London WC1E 6BT, UK. ${ }^{3}$ Department of Pharmaceutical Biosciences and Science for Life Laboratory, Uppsala University, 751 24 Uppsala, Sweden. ${ }^{4}$ Division of Computational Science and Technology, KTH, 10044 Stockholm, Sweden. ${ }^{5}$ Dept. Computer and Systems Sciences, Stockholm University, Box 7003, 16407 Kista, Sweden. ${ }^{6}$ MTM Research Centre, School of Science and Technology, Örebro University, 70182 Örebro, Sweden.

Received: 7 February 2021 Accepted: 10 April 2021

Published online: 29 April 2021

\section{References}

1. Yang H, Sun L, Li W, Liu G, Tang Y (2018) in silico prediction of chemical toxicity for drug design using machine learning methods and structural alerts. Front Chem 6:30. https://doi.org/10.3389/fchem.2018.00030

2. Klambauer G, Hochreiter S, Rarey M (2019) Machine learning in drug discovery. J Chem Inf Model 59(3):945-946. https://doi.org/10.1021/acs. jcim.9b00136

3. Vo AH, Van Vleet TR, Gupta RR, Liguori MJ, Rao MS (2020) An overview of machine learning and big data for drug toxicity evaluation. Chem Res Toxicol 33(1):20-37. https://doi.org/10.1021/acs.chemrestox.9b00227

4. Steger-Hartmann T, Boyer S (2020) Computer-based prediction models in regulatory toxicology. Regulatory toxicology. Springer, Berlin, pp 123-131. https://doi.org/10.1007/978-3-642-35374-1 36

5. Richard AM, Judson RS, Houck KA, Grulke CM, Volarath P, Thillainadarajah I, Yang C, Rathman J, Martin MT, Wambaugh JF, Knudsen TB, Kancherla J, Mansouri K, Patlewicz G, Williams AJ, Little SB, Crofton KM, Thomas RS (2016) ToxCast chemical landscape: paving the road to 21st century toxicology. Chem Res Toxicol 29(8):1225-1251. https://doi.org/10.1021/ acs.chemrestox.6b00135

6. Huang R, Sakamuru S, Martin MT, Reif DM, Judson RS, Houck KA, Casey W, Hsieh JH, Shockley KR, Ceger P, Fostel J, Witt KL, Tong W, Rotroff DM, Zhao T, Shinn P, Simeonov A, Dix DJ, Austin CP, Kavlock RJ, Tice RR, Xia M (2014) Profiling of the Tox21 10K compound library for agonists and antagonists of the estrogen receptor alpha signaling pathway. Sci Rep 4:1-9. https:// doi.org/10.1038/srep05664
7. Richard AM, Huang R, Waidyanatha S, Shinn P, Collins B, Thillainadarajah I, Grulke CM, Williams AJ, Lougee RR, Judson RS, Houck KA, Shobair M, Yang C, Rathman JF, Yasgar A, Fitzpatrick SC, Simeonov A, Thomas RS, Crofton KM, Paules RS, Bucher JR, Austin CP, Kavlock RJ, Tice RR (2020) The Tox21 10K compound library: collaborative chemistry advancing toxicology. Chem Res Toxicol. https://doi.org/10.1021/acs.chemrestox.0c00264

8. Cases M, Briggs K, Steger-Hartmann T, Pognan F, Marc P, Kleinöder T, Schwab CH, Pastor M, Wichard J, Sanz F (2014) The eTOX data-sharing project to advance in Silico drug-induced toxicity prediction. Int J Mol Sci 15(11):21,136-21,154. https://doi.org/10.3390/ijms151121136

9. Pastor M, Quintana J, Sanz F (2018) Development of an infrastructure for the prediction of biological endpoints in industrial environments. Lessons learned at the eTOX project. Front Pharmacol 9:1-8. https://doi.org/10. 3389/fphar.2018.01147

10. Golbraikh A, Shen M, Xiao Z, Xiao YD, Lee KH, Tropsha A (2003) Rational selection of training and test sets for the development of validated QSAR models. J Comput Aided Mol Des 2003(17):241-53. https://doi.org/10. 1023/A:1025386326946

11. Martin TM, Harten P, Young DM, Muratov EN, Golbraikh A, Zhu H, Tropsha A (2012) Does rational selection of training and test sets improve the outcome of QSAR modeling? J Chem Inf Model 52(10):2570-2578. https:// doi.org/10.1021/ci300338w

12. Leonard JT, Roy K (2006) On selection of training and test sets for the development of predictive QSAR models. QSAR Combinat Sci 25(3):235251. https://doi.org/10.1002/qsar.200510161

13. Andrada MF, Vega-Hissi EG, Estrada MR, Garro Martinez JC (2017) Impact assessment of the rational selection of training and test sets on the predictive ability of QSAR models. SAR QSAR Environ Res 28(12):1011-1023. https://doi.org/10.1080/1062936X.2017.1397056

14. Klimenko K, Rosenberg SA, Dybdahl M, Wedebye EB, Nikolov NG (2019) QSAR modelling of a large imbalanced aryl hydrocarbon activation dataset by rational and random sampling and screening of 80,086 REACH pre-registered and/or registered substances. PLoS ONE 14(3):1-21. https://doi.org/10.1371/journal.pone.0213848

15. Mathea M, Klingspohn W, Baumann K (2016) Chemoinformatic classification methods and their applicability domain. Mol Inf 35(5):160-180. https://doi.org/10.1002/minf.201501019

16. Hanser T, Barber C, Marchaland JF, Werner S (2016) Applicability domain: towards a more formal definition. SAR QSAR Environ Res 27(11):865-881. https://doi.org/10.1080/1062936X.2016.1250229

17. Bosnić Z, Kononenko I (2008) Comparison of approaches for estimating reliability of individual regression predictions. Data Knowl Eng 67(3):504-516. https://doi.org/10.1016/j.datak.2008.08.001

18. Aniceto N, Freitas AA, Bender A, Ghafourian T (2016) A novel applicability domain technique for mapping predictive reliability across the chemical space of a QSAR: Reliability-density neighbourhood. J Cheminf 8(1):1-20. https://doi.org/10.1186/s13321-016-0182-y

19. Dragos H, Gilles M, Alexandre V (2009) Predicting the predictability: a unified approach to the applicability domain problem of QSAR models. J Chem Inf Model 49(7):1762-1776. https://doi.org/10.1021/ci9000579

20. Sheridan RP (2015) The relative importance of domain applicability metrics for estimating prediction errors in QSAR varies with training set diversity. J Chem Inf Model 55(6):1098-1107. https://doi.org/10.1021/acs. jcim.5b00110

21. Alves VM, Muratov EN, Zakharov A, Muratov NN, Andrade $\mathrm{CH}$, Tropsha A (2018) Chemical toxicity prediction for major classes of industrial chemicals: Is it possible to develop universal models covering cosmetics, drugs, and pesticides? Food Chem Toxicol 112:526-534. https://doi.org/ 10.1016/j.fct.2017.04.008

22. Cortés-Ciriano I, Škuta C, Bender A, Svozil D (2020) QSAR-derived affinity fingerprints (part 2): modeling performance for potency prediction. J Cheminf 12(1):1-17. https://doi.org/10.1186/s13321-020-00444-5

23. Vovk V (2013) Conditional validity of inductive conformal predictors. Mach Learn 92(2-3):349-376. https://doi.org/10.1007/s10994-013-5355-6

24. Vovk V, Gammerman A, Shafer G (2005) Algorithmic learning in a random world. Springer Science \& Business Media, Berlin

25. Fedorova V, Gammerman A, Nouretdinov I, Vovk V (2012) Plug-in martingales for testing exchangeability on-line. In: Proceedings of the 29th international conference on machine learning, ICML 2012 2. pp 1639-1646 
26. Morger A, Mathea M, Achenbach JH, Wolf A, Buesen R, Schleifer KJ, Landsiedel R, Volkamer A (2020) KnowTox: pipeline and case study for confident prediction of potential toxic effects of compounds in early phases of development. J Cheminf 12(1):1-17. https://doi.org/10.1186/ s13321-020-00422-x

27. Huang Ruili, Xia Menghang (2017) Editorial: Tox21 challenge to build predictive models of nuclear receptor and stress response pathways as mediated by exposure to environmental toxicants and drugs. Front Environ Sci 5(3):5. https://doi.org/10.1038/ncomms

28. NIH_National Center for Advancing Translational Sciences (2014) Tox21 data challenge. https://ripod.nih.gov/tox21/challenge/data.jsp

29. Atkinson FCGEE (2014) Standardiser. https://github.com/flatkinson/stand ardiser

30. Heller SR, McNaught A, Pletnev I, Stein S, Tchekhovskoi D (2015) InChl, the IUPAC international chemical identifier. J Cheminf. https://doi.org/10. 1186/s13321-015-0068-4

31. Huang R, Xia M, Nguyen DT, Zhao T, Sakamuru S, Zhao J, Shahane SA, Rossoshek A, Simeonov A (2016) Tox21 challenge to build predictive models of nuclear receptor and stress response pathways as mediated by exposure to environmental chemicals and drugs. Front Environ Sci 3:1-9. https://doi.org/10.3389/fenvs.2015.00085

32. Faulon JL, Visco DP, Pophale RS (2003) The signature molecular descriptor. 1. Using extended valence sequences in QSAR and QSPR studies. J Chem Inf Comput Sci 43(3):707-720. https://doi.org/10.1021/ci020345w

33. Faulon JL, Collins MJ, Carr RD (2004) The signature molecular descriptor. 4. Canonizing molecules using extended valence sequences. J Chem Inf Comput Sci 44(2):427-436. https://doi.org/10.1021/ci0341823

34. Aros Bio (2020) CPSign. https://arosbio.com/cpsign/

35. Kensert A, Alvarsson J, Norinder U, Spjuth O (2018) Evaluating parameters for ligand-based modeling with random forest on sparse data sets. J Cheminf 10(1):1-10. https://doi.org/10.1186/s13321-018-0304-9

36. Alvarsson J, Eklund M, Engkvist O, Spjuth O, Carlsson L, Wikberg JE, Noeske T (2014a) Ligand-based target prediction with signature fingerprints. J Chem Inf Model 54(10):2647-2653. https://doi.org/10.1021/ci500 $361 \mathrm{u}$

37. Alvarsson J, Eklund M, Andersson C, Carlsson L, Spjuth O, Wikberg JE (2014b) Benchmarking study of parameter variation when using signature fingerprints together with support vector machines. J Chem Inf Model 54(11):3211-3217. https://doi.org/10.1021/ci500344v

38. Sun J, Carlsson L, Ahlberg E, Norinder U, Engkvist O, Chen H (2017) Applying Mondrian cross-conformal prediction to estimate prediction confidence on large imbalanced bioactivity data sets. J Chem Inf Model 57(7):1591-1598. https://doi.org/10.1021/acs.jcim.7b00159
39. Toccaceli P, Gammerman A (2019) Combination of inductive Mondrian conformal predictors. Mach Learn 108(3):489-510. https://doi.org/10. 1007/s10994-018-5754-9

40. Alvarsson J, Arvidsson McShane S, Norinder U, Spjuth O (2021) Predicting with confidence: using conformal prediction in drug discovery. J Pharm Sci 110(1):42-49. https://doi.org/10.1016/j.xphs.2020.09.055

41. Norinder U, Carlsson L, Boyer S, Eklund M (2014) Introducing conformal prediction in predictive modeling. A transparent and flexible alternative to applicability domain determination. J Chem Inf Model 54(6):15961603. https://doi.org/10.1021/ci5001168

42. Linusson $H$, Norinder $U$, Boström $H$, Johansson U, Löfström T (2017) On the calibration of aggregated conformal predictors. In: Proceedings of the sixth workshop on conformal and probabilistic prediction and applications 60. pp 154-173

43. Carlsson L, Eklund M, Norinder U, Carlsson L, Eklund M, Norinder U, Conformal A, Lazaros P, Maglogiannis I, Papadopoulos H, Sioutas S, Ifip CM (2014) Aggregated conformal prediction. In: IFIP advances in information and communication technology. pp 231-240

44. Gauraha N, Spjuth O (2018) Synergy conformal prediction. DiVA preprint 360504. http://urn.kb.se/resolve?urn=urn:nbn:se:uu:diva-360504

45. Pedregosa F, Varoquaux G, Gramfort A, Michel V, Thirion B, Grisel O, Blondel M, Prettenhofer P, Weiss R, Dubourg V, Vanderplas J, Passos A, Cournapeau D, Brucher M, Perrot M, Duchesnay E (2011) Scikit-learn: machine learning in python. J Mach Learn Res 12:2825-2830

46. Linusson H (2015) Nonconformist. http://donlnz.github.io/nonconform ist/

47. Gauraha N (2018) Synergy conformal prediction code. https://github. com/niha21/scp_code

48. Attene-Ramos MS, Miller N, Huang R, Michael S, Itkin M, Kavlock RJ, Austin CP, Shinn P, Simeonov A, Tice RR, Xia M (2013) The Tox21 robotic platform for the assessment of environmental chemicals - from vision to reality. Drug Discov Today 18(15-16):716-723. https://doi.org/10.1016/j.drudis. 2013.05.015.

49. Azadeh M, Sondag P, Wang Y, Raines M, Sailstad J (2019) Quality controls in ligand binding assays: recommendations and best practices for preparation, qualification, maintenance of lot to lot consistency, and prevention of assay drift. AAPS J. https://doi.org/10.1208/s12248-019-0354-6

\section{Publisher's Note}

Springer Nature remains neutral with regard to jurisdictional claims in published maps and institutional affiliations.
Ready to submit your research? Choose BMC and benefit from:

- fast, convenient online submission

- thorough peer review by experienced researchers in your field

- rapid publication on acceptance

- support for research data, including large and complex data types

- gold Open Access which fosters wider collaboration and increased citations

- maximum visibility for your research: over $100 \mathrm{M}$ website views per year

At BMC, research is always in progress.

Learn more biomedcentral.com/submissions 